\title{
JOURNAL.RU
}

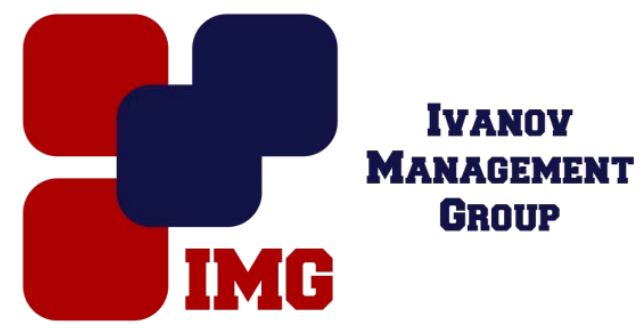

Смирнов А.В., Андрианов И.А., Суконщиков А.А., Бахтенко Е.А. Вологодский государственный университет

Вологда, Россия

doi: 10.18411/lj-31-01-2017-1-13

idsp 000001:lj-31-01-2017-1-13

\section{Математическая модель логистического управления циклом заказа с использованием факторного анализа и механизмов прогнозирования}

Для увеличения надежности выполнения заказа за счет уменьшения цикла заказа предлагается внедрить в логистическую информационную систему математическую модель расчета коэффициента прогноза выполнения заказа Хпрогни коэффициента надежности выполнения заказа Qнад.

Модель реализуется следующим образом - информационной системе необходимо провести выборку факторов времени Xn и факторов надежности $\mathrm{Qn}$, произвести анализ и выдать данные о планируемом (допустимом) времени выполнения заказа T.

В зависимости от требования, предъявляемого клиентомк выполнению заказа, производится расчет среднего времени выполнения заказаХср. Например, при выборе клиентом приоритета стоимости товара Sзак информационная система определяет наиболее рациональное место отгрузки товара с учетом минимальногоSзак, выбрав из базы данных случаи, по которым товар был доставлен из планируемого места поставки, и рассчитывает X ср.

В общем виде уравнение расчета среднего времени заказа представлено в виде:

$$
X_{c p}=\frac{\sum_{i=1}^{n} X_{c л i}}{N_{c l}},
$$

где Хср - среднее время выполнения заказа;

Хслі - время, выполнения і - й логистической операции;

Nсл- общее количество случаев.

В ряде работ для оценки изменения среднего квадратического отклонениябТ используется формула: 


$$
\sigma_{T}=\sqrt{\sum_{i=1}^{N} \sigma_{i}^{2}+2 \sum_{i \leq j} r_{i j}} \sigma_{i} \sigma_{j},
$$

где $\sigma_{i}$ - среднее квадратическое отклонение времени выполнения i -й операции логистического цикла;

$r_{i j}$ - коэффициент корреляции между і -й и ј -й операциями цикла.

Однако формула (2) недостаточна для выявления причин, влияющих на отклонения планируемого времени выполнения заказа.

Для повышения обоснованности планируемого времени выполнения заказа предлагается формула:

$$
T=X_{c p} \times X_{\text {прогн }} \times Q_{\text {над }},
$$

где $\mathrm{T}-$ планируемое время выполнения заказа;

Хср - среднее время выполнения заказа;

Хпрогн- коэффициент прогноза выполнения заказа;

Qнад- коэффициент надежности выполнения заказа.

На основании анализа времени выполнения заказов и влияющих на них факторов, полученных из информационной базы данных, применяя метод корреляционно-регрессионного анализа, автором были выявлены факторы, воздействующие на цикл заказа.

Для вычисления Хпрогн и Qнадпредставлены ключевые факторы времени цикла заказа в розничной торговле КТ (рис. 1).

В результате факторного анализа получено уравнение для расчета Хпрогн , имеющее вид:

$$
\begin{aligned}
& X_{\text {проги }}=1,0599+2,459 \times X 1+1,641 \times X 2+1,2166 \times X 3+0,832 \times X 4+ \\
& +0,335 \times X 5+0,9988 \times X 6+0,754 \times X 7+1,448 \times X 8+1,9404 \times X 9+ \\
& +1,641 \times X 11+1,2166 \times X 12+0,832 \times X 13+0,335 \times X 14+0,9988 \times X 15
\end{aligned}
$$

Факторы X10 и X16 математической моделью не учитываются, так как они не оказывают значительного влияния на результативный признак. 


\begin{tabular}{|c|c|c|c|}
\hline Факторы & Описание факторов времени & Факторы & Описание факторов надежности \\
\hline $\mathrm{X} 1$ & Сезонность & \multirow{2}{*}{\multicolumn{2}{|c|}{ Количество брака на 1000 ед. товаров }} \\
\hline \multicolumn{2}{|c|}{$\begin{array}{c}\text { Время, затрачиваемое клиентом } \\
\text { на подачу заявки }\end{array}$} & & \\
\hline $\mathrm{X} 2$ & используя интернет-магазин & $\mathrm{X} 17$ & категории «A» \\
\hline $\mathrm{X} 3$ & используя терминал & $\mathrm{X} 18$ & категории «B» \\
\hline $\mathrm{X} 4$ & при помощи консультанта & $\mathrm{X} 19$ & категории «С» \\
\hline \multirow{2}{*}{\multicolumn{2}{|c|}{$\begin{array}{l}\text { Время, затрачиваемое } \\
\text { на обработку заявки }\end{array}$}} & & \\
\hline & & $\mathrm{X} 20$ & ошибка менеджера \\
\hline $\mathrm{X} 5$ & используя интернет-магазин & $\mathrm{X} 21$ & возврат товаров \\
\hline $\mathrm{X} 6$ & используя терминал & \multirow{2}{*}{\multicolumn{2}{|c|}{ Задержки поставки заказа }} \\
\hline $\mathrm{X} 7$ & при помощи консультанта & & \\
\hline \multirow{2}{*}{\multicolumn{2}{|c|}{$\begin{array}{l}\text { Время, затрачиваемое } \\
\text { клиентом на оплату }\end{array}$}} & $\mathrm{X} 22$ & не более 3 часов \\
\hline & & $\mathrm{X} 23$ & более 3 часов \\
\hline $\mathrm{X} 8$ & с использованием кредита & $\mathrm{X} 24$ & более 24 часов \\
\hline X9 & наличными средствами & & \\
\hline $\mathrm{X} 10$ & электронным способом & $\mathrm{X} 25$ & сбои информационной системы \\
\hline \multirow{2}{*}{\multicolumn{2}{|c|}{$\begin{array}{l}\text { Время, затрачиваемое } \\
\text { на комплектацию }\end{array}$}} & $\mathrm{X} 26$ & ошибки поставщиков \\
\hline & & & \\
\hline $\mathrm{X} 11$ & товаров категории «A» & & \\
\hline $\mathrm{X} 12$ & товаров категории «В» & & \\
\hline $\mathrm{X} 13$ & товаров категории «С» & & \\
\hline \multicolumn{2}{|c|}{$\begin{array}{c}\text { Время, затрачиваемое } \\
\text { на доставку заказов клиенту }\end{array}$} & & \\
\hline $\mathrm{X} 14$ & поставщиками через РЦ & & \\
\hline $\mathrm{X} 15$ & $\begin{array}{c}\text { распределительными } \\
\text { центрами }\end{array}$ & & \\
\hline $\mathrm{X} 16$ & $\begin{array}{c}\text { транспортной службой } \\
\text { магазина }\end{array}$ & & \\
\hline
\end{tabular}

Рис. 1. - Ключевые факторы времени и факторы надежности в цикле заказа розничной торговли

Для Qнадуравнение имеет вид:

$Q_{\text {над }}=1,11+(-9 \times X 17-39 \times X 18-17 \times X 19-16,3 \times$

$\times X 21-9 \times X 22-39 \times \times X 23-17 \times X 24-16,3 \times X 25) / 100$

Факторы X20 и Х26 математической моделью не учитываются, так как они также не оказывают значительного влияния на результативный признак при регрессионном анализе.

В целях рационализации выполнения логистических операций по реализации заказов предлагаются методические рекомендации по определению, на основе статистического моделирования, допустимого времени выполнения заказа, дифференцированного по каждой операции логистического процесса 
заказа, и использованию допустимого (планируемого) времени выполнения заказа (Т), с которым сравнивается фактическое время.

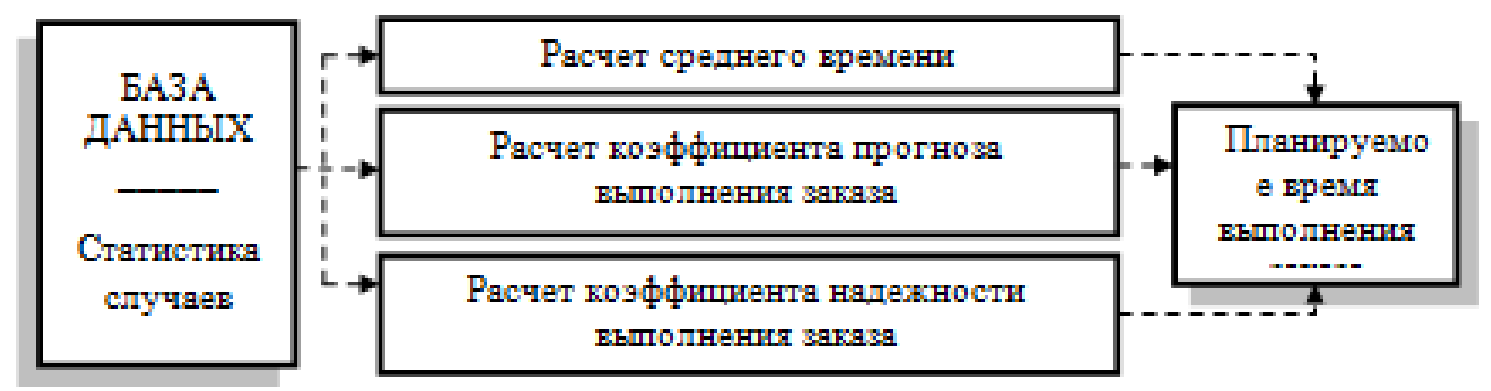

Рис. 2. - Схема расчета планируемого времени выполнения заказа

При выявленных отклонениях от допустимого времени выполнения заказа определяется зона ответственности, по вине которой произошло это отклонение, выявляются причины и принимаются управленческие решения. Например, для доставки товара на склад одного из РЦ в среднем уходит 2,5 часа. Фактически для выполнения данной операции потребовалось 3,6 часа. В результате статистического моделирования и расчета Тустановлено допустимое время 4 часа. При превышении времени информационная система устанавливает, по вине какой зоны ответственности это произошло, то есть, на какой логистической операции. Информация поступает в систему логистического менеджмента компании, который выявляет причины отклонений и принимает меры по повышению надежности выполнения заказа. 